\title{
COLD COLLAPSE AS A WAY OF MAKING ELLIPTICAL GALAXIES
}

\author{
L.A. Aguilar \\ Harvard-Smithsonian Center for Astrophysics \\ 60 Garden St., Cambridge MA 02138 USA \\ D. Merritt and M. Duncan \\ Canadian Institute for Theoretical Astrophysics \\ University of Toronto, Toronto ON M5S 1A1 CANADA
}

\section{SUMMARY}

We investigate whether dissipationless collapse starting from very cold, nonrotating initial conditions can produce objects resembling real elliptical galaxies. We also study the effect of various initial geometries on the shape of the final object. Collapses that are initially very cold $(2 T / W<0.1)$ are different from warmer collapses, due to the presence of a dynamical instability associated with clumping of nearly-radial orbits (Polyachenko 1981). This instability can produce very elongated bars (1.6 to 2.1 axis ratio) from spherical initial conditions. The instability is also present in models evolved from oblate and triaxial initial conditions. Warm collapses tend to preserve their initial shapes. Cold initial conditions produce objects whose surface density profiles are well fit by a de-Vaucouleurs law; warm collapses, on the other hand, produce a core-halo profile. A large collapse factor seems necessary to produce objects resembling real galaxies; the same collapse factor guarantees the presence of the radial orbit instability. It thus appears that initial flattening is not crucial for producing prolate or nearly prolate galaxies. Oblate galaxies, on the contrary, seem very difficult to form, unless extremely flattened initial conditions are invoked. Preliminary experiments suggest that these results are not changed by realistic amounts of angular momentum.

\section{NUMERICAL EXPERIMENTS}

Our initial conditions have a $\rho \propto r^{-1}$ density profile and Gaussian thermal motion independent of position. Some of the spherical models are squeezed along one or two axes to obtain oblate or triaxial initial conditions. All the models have the same binding energy. We used an $\mathrm{N}$-body code that approximates the forces by an expansion in spherical harmonics up to octupolar terms (Aguilar and White 1985). All our runs use 5000 particles and are followed during 10 free-fall times at the initial half mass radius. Several of our experiments have been checked with a direct-summation Aarseth code. The two codes yield results that are in good agreement. We have run collapses with initial virial ratios $2 T / W$ ranging from 0.3 to 0.01 , and oblate initial conditions ranging from spherical to an axis ratio of 2.5 to 1 . One experiment was realized with 1:2:3 triaxial initial conditions. 


\section{RESULTS}

a) Shapes. Table 1 shows the eigenvalues of the inertia tensor for the $80 \%$ most bound particles of the final configurations, which we used as estimators of the degree of flattening.

\section{TABLE I}

Final axis ratio as a function of initial temperature and axis ratio

\begin{tabular}{|c|c|c|c|c|c|c|c|}
\hline $\log 2 T / h$ & $=-0.50$ & -0.75 & -1.00 & -1.25 & -1.50 & -1.75 & -2.00 \\
\hline$k=1.0$ & $1 \mathrm{a}-1 \mathrm{O} 0 \mathrm{l}$ & $101+100$ & $1,611-1$ & 1.8121 & $1,8-1,-1$ & $2,01,2-1$ & $2,1-1,3-1$ \\
\hline 1.5 & $1.2,1,1.1$ & $1.2-1.1-1$ & $(6-1 / 1)$ & $1,91,21$ & $1.6-1.0-1$ & $1.8-1.0-1$ & \\
\hline 2.0 & $1.4+4.4$ & $1.7-1.4-1$ & $1.9-1.4-1$ & $1.9-1.3-1$ & $2.2-13-1$ & 19121 & \\
\hline 2.5 & & 1.8 .1 .5 .1 & 2.1-1.5-1 & 2.3-1.5-1 & 2.11 .31 & 2.31 .41 & \\
\hline $1: 2: 3$ & & & & & $2.1-1.21$ & & \\
\hline & & & $>$ & Bar Ins & ility & & \\
\hline & Solder & & Brats: & Prolate & Triaxial & & \\
\hline
\end{tabular}

Notice the abrupt onset of non-sphericity for initially spherical models colder than $2 T / W \approx 0.1$; the final axis ratio is a slowly varying function of temperature afterwards. The same qualitative behavior is presented by experiments started from non-spherical initial conditions; but a gap of triaxial models now appears at the boundary of the bar instability and widens as we increase the initial flattening.

b) Surface density profiles. The surface density profiles of the final models have been obtained using elliptical annuli with the same axis ratio as the inertia tensor. The results are insensitive to the degree of initial flattening and are a good fit to the de Vaucouleurs law only when $2 T / W \leq 0.1$.

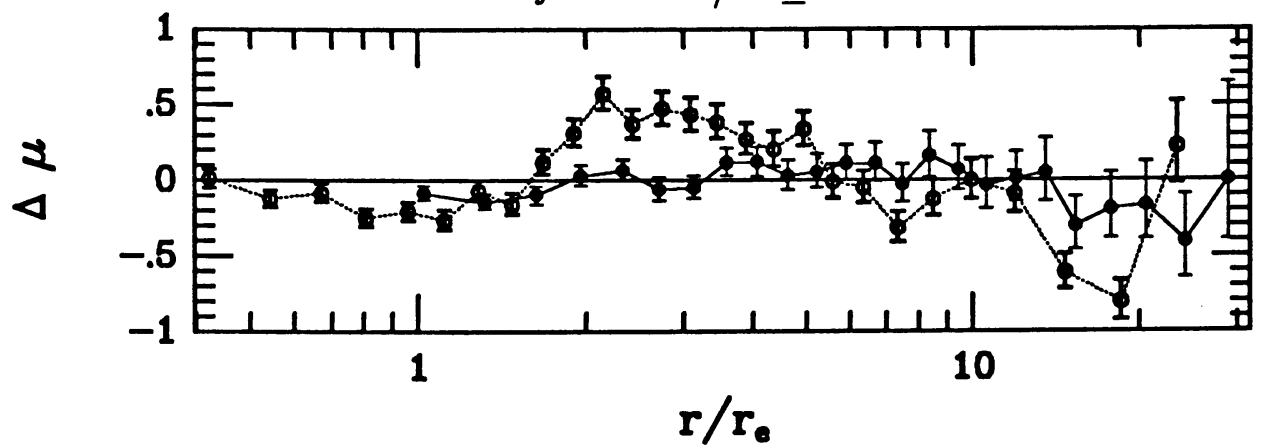

Figure 1. Residuals (in magnitudes) of fits to $r^{1 / 4}$-laws for a warm (dotted line) and cold (solid line) collapse $(2 T / W=0.32,0.02)$. The error bars are the expected statistical fluctuations due to the number of particles in each bin.

REFERENCES

Aguilar, L.A. and White, S.D.M. 1985. Astrophys. J., 295, 374.

Polyachenko, V.L. 1981. Pis'ma Astr. Zh., 7, 142 (translated in Soviet Astr. Lett., 7, 79). 